\title{
Further Discussion of the Specific Polymerization Behavior of Triallyl I socyanurate. Enhanced Occurrence of Intermolecular Crosslinking at an Early Stage of Polymerization
}

\author{
Akira Matsumoto, ${ }^{\dagger}$ Chikaomi HiRao, Kazuyoshi Miyata, Hiroyuki Aota, \\ Yoshihiro TAKAYAMA, ${ }^{*}$ and Hiroyuki TORIDOME* \\ Department of Applied Chemistry, Faculty of Engineering and High Technology Research Center, \\ Kansai University, Suita, Osaka 564-8680, Japan \\ *Nippon Kasei Chemical Co., Ltd, 34 Takayama, Onahama, Iwaki-shi, Fukushima 971-8101, Japan
}

(Received March 22, 2002; Accepted June 3, 2002)

\begin{abstract}
KEY WORDS Triallyl Isocyanurate / Diallyl Terephthalate / Steric Effect / Intermolecular Crosslinking / Thermodynamic Excluded Volume Effect / Molecular Weight Distribution /
\end{abstract}

As part of our continuing studies concerned with the free-radical polymerizations of multiallyl compounds in terms of allyl polymerization, cyclopolymerization, and gelation, ${ }^{1-3}$ we have been concerned with the elucidation of the specific polymerization behavior of triallyl isocyanurate (TAIC), especially focusing on the steric effect caused by the bulky isocyanurate side groups. ${ }^{4-9}$ Thus, it was found by chance that the primary chain length of the TAIC polymer is quite high compared with the polymerization of its isomer triallyl cyanurate (TAC), being ascribed to the reduced monomer chain transfer (degradative chain transfer characteristic of allyl polymerization $)^{10}$ due to the steric effect by the bulky isocyanurate side groups on transition state formation. ${ }^{4}$ This steric effect is further discussed by both the copolymerization with allyl benzoate and the telomerization in the presence of $\mathrm{CBr}_{4},{ }^{5}$ by the polymerization of diallyl alkyl isocyanurates, ${ }^{6}$ by the polymerization and copolymerization of trimethallyl isocyanurate and trimethallyl cyanurate, ${ }^{7}$ and by the copolymerization with sterically unhindered monomers. ${ }^{8}$ In particular, the last two papers ${ }^{7,8}$ are concerned with the discussion of the steric effect on the reactivity of growing polymer radical in terms of the sequence length dependence of the sterically bulky TAIC units. This is extended to the non-terminal units effect on the cyclopolymerization of TAIC. ${ }^{9}$

As our further pursuit of the specific polymerization behavior of TAIC in comparison with other multiallyl compounds, the molecular-weight distribution (MWD) curves of resulting prepolymers were examined by using size-exclusion chromatography (SEC) equipped

${ }^{\dagger}$ To whom correspondence should be addressed. with a multiangle laser light scattering (MALLS) device (SEC-MALLS) in order to explore in more detail the occurrence of intermolecular crosslinking reaction. Here it is worthwhile to note that SEC is commonly used to determine the molecular weight and the MWD of resulting prepolymers. However, as a technique it is not always applicable to our crosslinking polymerization systems because the structure of prepolymer changes from a linear to a branched form with progressing polymerization. On the other hand, SEC-MALLS is expected to be a very useful and efficient tool in characterizing our prepolymers because the use of MALLS, as an absolute detector, enables the molecular weight and MWD to be determined directly without any calibration. ${ }^{11}$

Thus, this article deals with the further discussion of the specific polymerization behavior of TAIC; the occurrence of intermolecular crosslinking reaction was explored in detail as compared with the polymerization behavior of diallyl terephthalate (DAT) as a typical multiallyl compound. In the polymerization of TAIC, the conversion dependence of the MWD profiles of resulting prepolymers was different from that in DAT polymerization, i.e., the MWD curves were broadened toward a higher molecular weight side at an earlier stage of polymerization as a reflection of the occurrence of intermolecular crosslinking reaction. This enhanced occurrence of intermolecular crosslinking may be caused by the rigidity of TAIC polymer chain having bulkier isocyanurate side groups than the polymer chains derived from the polymerizations of other multiallyl compounds; the rigid TAIC polymer chain leads to a reduced interaction between polar isocyanuric rings 
belonging to both polymer chain and TAIC monomer and thus, TAIC monomer may act as a poor solvent for the resulting TAIC prepolymers. In a poor solvent, the significance of the thermodynamic excluded volume effect $^{2}$ for preventing the intermolecular crosslinking between the growing polymer radical and prepolymer, especially at high molecular weight, would be lessened.

\section{EXPERIMENTAL}

TAIC and allyl dipropyl isocyanurate (ADPIC), supplied by Nippon Kasei Chemical Co., Ltd., and DAT, supplied by Daiso Co., Ltd., as monomers were purified by vacuum distillation under nitrogen. 2,2'Azobisisobutyronitrile (AIBN) as initiator was purified by recrystallization from methanol.

Polymerizations were carried out in ampoules as described previously. ${ }^{12}$ After the desired time, the reaction mixture was poured into a large amount of hexane to precipitate the polymer.

The weight-average molecular weight, $\bar{M}_{\mathrm{w}}$, and MWD were measured by SEC using a dual detector system, set in the direction of flow, consisting of a MALLS device and a differential refractometer in sequence. SEC-MALLS measurements were carried out at $40^{\circ} \mathrm{C}$ in tetrahydrofuran using a Shodex GPC KF$806 \mathrm{~L} \times 5$ columns, at polymer concentrations $0.1-0.5 \%$ $(\mathrm{w} / \mathrm{v})$ and a flow rate of $1 \mathrm{~mL} \mathrm{~min}^{-1}$. The MALLS device was a DAWN DSP (Wyatt Technology Corp.) where the laser beam (wavelength: $632.8 \mathrm{~nm}$ ) was focused on a $67 \mu \mathrm{L}$ flow cell.

\section{RESULTS AND DISCUSSION}

Specific Conversion-Dependency of MWD Profiles of TAIC Prepolymers Obtained at an Early Stage of Polymerization as Compared with DAT Polymerization

The mechanistic elucidation of network formation in free-radical crosslinking polymerization and copolymerization of multivinyl compounds, being a long-term controversial problem because of the complexity of the reaction involved, has been discussed ${ }^{2}$ on the basis of the comparison of the experimental results with FloryStockmayer gelation theory, ${ }^{13}$ which was developed later by Gordon ${ }^{14}$ to predict the conversion at gelation for a polymerizing monomer which undergoes cyclization.

Thus, in the bulk polymerizations of multiallyl compounds the gelled polymers are usually obtained above $20 \%$ conversion, e.g., the gel points are estimated to be $23.5,22.0$, and $21.3 \%$, respectively, for DAT, ${ }^{15}$ triallyl trimellitate, ${ }^{16}$ and TAC $^{4}$ polymerizations. Furthermore, the experimental results are quite high in com-

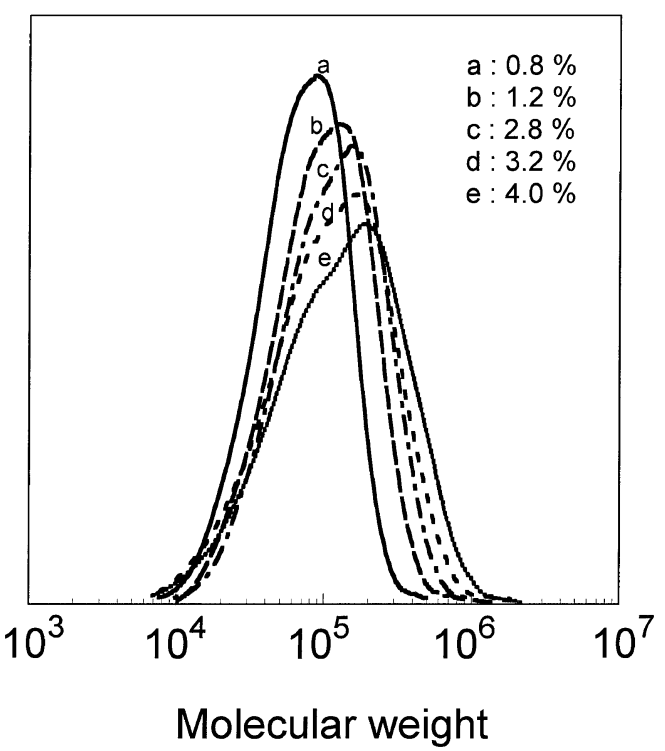

Figure 1. Variation of MWD curves with conversion for the bulk polymerization of TAIC using $0.01 \mathrm{~mol} \mathrm{~L}^{-1}$ of AIBN at $60^{\circ} \mathrm{C}$.

parison with the theoretical gel points calculated from Gordon's equation as exemplified by DAT polymerization. ${ }^{17,18}$ In this connection, the thermodynamic excluded volume effect on the intermolecular crosslinking reaction between the growing polymer radical and prepolymer would be significant as a primary factor for the greatly delayed gelation from theory, considering that each monomer is a good solvent for the corresponding prepolymers produced. ${ }^{2,19}$ That is, the intermolecular crosslinking reaction between the growing polymer radical and prepolymer, especially at high molecular weight, is suppressed at an early stage of polymerization, since the high molecular weight prepolymer concentration is quite low. In this work, we tried to pursue in more detail the occurrence of intermolecular crosslinking in the polymerization of TAIC, especially focusing on the thermodynamic excluded volume effect at an early stage of polymerization. That the gelation occurs at $12.4 \%$ conversion in the polymerization of TAIC ${ }^{4}$ is quite different from the polymerizations of common multiallyl compounds where gelation occurred at more than $20 \%$ conversion.

Bulk polymerizations of TAIC and DAT were carried out using $0.01 \mathrm{~mol} \mathrm{~L}^{-1}$ of AIBN at $60^{\circ} \mathrm{C}$. Figure 1 shows the variation of MWD curves with conversion at an early stage of polymerization in TAIC polymerization; the MWD profiles were broadened toward a higher molecular weight side with conversion, clearly demonstrating the occurrence of intermolecular crosslinking even at $1.2 \%$ conversion. On the other hand, in DAT polymerization almost no occurrence of intermolecular crosslinking was observed at an early stage of polymerization as shown in Figure 2, since even at $4.0 \%$ conversion MWD profiles were only a bit 


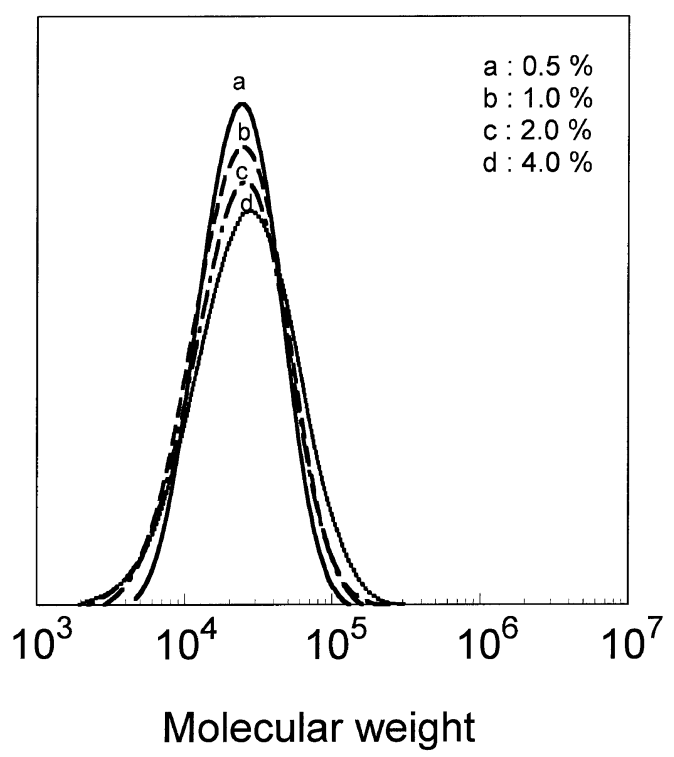

Figure 2. Variation of MWD curves with conversion for the bulk polymerization of DAT using $0.01 \mathrm{~mol} \mathrm{~L}^{-1}$ of AIBN at $60^{\circ} \mathrm{C}$.

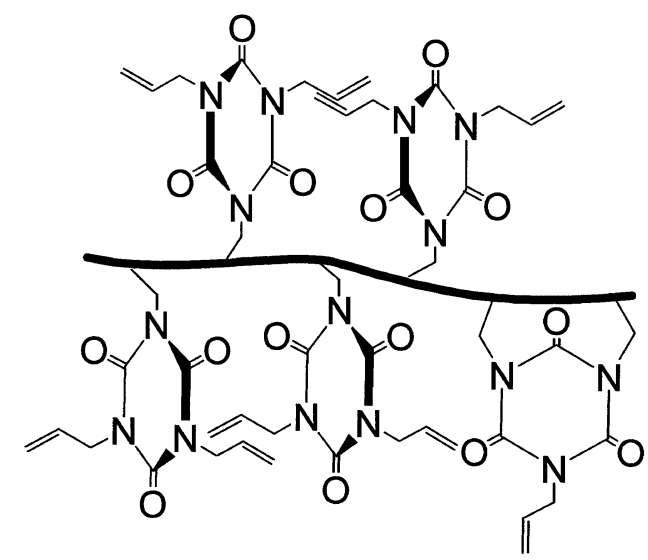

Figure 3. TAIC polymer chain having bulky isocyanate side groups.

broadened toward a higher molecular weight side.

\section{Mechanistic Discussion}

The detailed comparison of both TAIC and DAT polymerization results clearly demonstrates that in the polymerization of TAIC the intermolecular crosslinking reaction occurred at a much earlier stage of polymerization compared with DAT polymerization, although the results shown in Figure 2 are common for the polymerizations of multiallyl compounds. ${ }^{2}$ This specific polymerization behavior of TAIC may correlate with a less significance of the thermodynamic excluded volume effect mentioned above. ${ }^{2,19}$ That is, as is depicted in Figure 3, TAIC polymer chain having bulky isocyanurate side groups, in which only one methylene unit intervenes between the polymer chain and the isocyanuric ring, would be more rigid than the polymer chains derived from the polymerizations of other multiallyl compounds. Thus, an interaction between TAIC

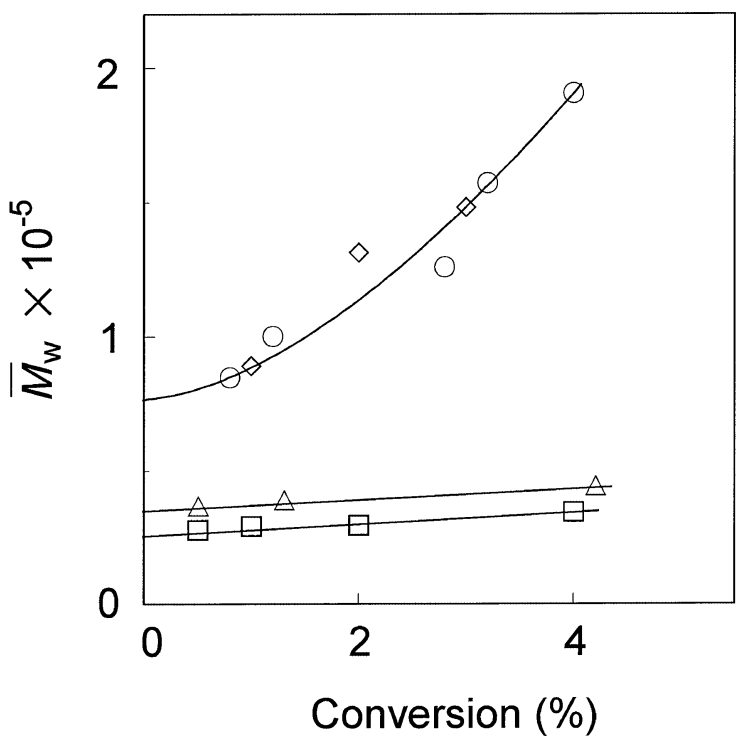

Figure 4. Dependence of $\bar{M}_{\mathrm{w}}$ on conversion (see Figures 1 and 2): (○) TAIC homopolymerization; $(\triangle)$ TAIC/DAT (50/50 $\mathrm{mol} / \mathrm{mol}$ ) copolymerization; ( $\square)$ DAT homopolymerization; $(\diamond)$ TAIC/ADPIC (50/50 mol/mol) copolymerization.

polymer chain having bulky isocyanurate side groups and TAIC monomer could be quite weakened by sterically suppressed access of TAIC monomer to bulky isocyanurate side groups of TAIC polymer chain. On the contrary, an interaction between TAIC monomers is quite high, as is reflected on the high melting point of $24^{\circ} \mathrm{C}$, because a symmetrical TAIC monomer contains a polar isocyanuric ring having three carbonyl groups. As a result, TAIC monomer would not act as a good solvent for TAIC prepolymer, being different from common cases of the bulk polymerizations of vinyl monomers. Therefore, the thermodynamic excluded volume effect on the intermolecular crosslinking reaction between the growing polymer radical and prepolymer, as a primary factor for the greatly delayed gelation from theory, ${ }^{2}$ would be less significant. This may lead to an enhanced occurrence of intermolecular crosslinking at an earlier stage of polymerization.

As an extension of the above discussion, TAIC was copolymerized with $50 \mathrm{~mol} \%$ of DAT. Figure 4 shows the dependence of molecular weights of resulting precopolymers on conversion, along with the results of both TAIC and DAT homopolymerizations. Obviously, the molecular weight decreased by the copolymerization with DAT as a reflection of reduced occurrence of intermolecular crosslinking. By assuming equal reactivity of all allyl groups belonging to both TAIC and DAT, the composition of resulting TAIC/DAT prepolymer obtained at an early stage of polymerization would be TAIC:DAT $=3: 2$ unit mol ratio and thus, the steric crowding of TAIC polymer chain by the sequential link of bulky isocyanurate side groups would be lessened 
by the incorporation of DAT units into the polymer chain. Thus, an interaction between TAIC/DAT prepolymer chain and both monomers could be significant in TAIC/DAT copolymerization as compared with the weakened interaction in TAIC homopolymerization by sterically suppressed access of TAIC monomer to bulky isocyanurate side groups of TAIC polymer chain. On the other hand, the bulky uncyclized TAIC units could be introduced into the polymer chain by the copolymerization of TAIC with ADPIC as a mono-ene counterpart of TAIC. The dependence of molecular weights of resulting precopolymers on conversion for TAIC/ADPIC $(50 / 50 \mathrm{~mol} / \mathrm{mol})$ copolymerization was shown in Figure 4; its dependency was observed to be similar to TAIC homopolymerization in conformity with our expectation.

\section{REFERENCES}

1. M. Oiwa and A. Matsumoto, "Progress in Polymer Science Japan”, Kodansha, Tokyo, 1974, vol.7, p 107.

2. A. Matsumoto, Adv. Polym. Sci., 123, 41 (1995).

3. A. Matsumoto, Prog. Polym. Sci., 26, 189 (2001).

4. A. Matsumoto, H. Inoue, T. Matsumoto, M. Oiwa, T. Kimura, and H. Saito, J. Macromol. Sci., Chem., A26, 1279 (1989).

5. A. Matsumoto, T. Matsumoto, H. Inoue, M. Oiwa, and H. Saito, Eur. Polym. J., 26, 661 (1990).

6. A. Matsumoto, K. Watanabe, T. Matsumoto, H. Aota, M.
Hirabayashi, A. Kameyama, and T. Nakanishi, J. Macromol. Sci., Pure Appl. Chem., A35, 1889 (1998).

7. A. Matsumoto, F. Hirai, Y. Sumiyama, H. Aota, Y. Takayama, A. Kameyama, and T. Nakanishi, Eur. Polym. J., 35195 (1999).

8. A. Matsumoto, H. Yamasaki, M. Katsumoto, H. Aota, Y. Takayama, A. Kameyama, and T. Nakanishi, Eur. Polym. J., 36, 1741 (2000).

9. A. Matsumoto, K. Watanabe, H. Aota, Y. Takayama, A. Kameyama, and T. Nakanishi, Polymer, 41, 3883 (2000).

10. P. D. Bartlett and R. Altschul, J. Am. Chem. Soc., 67, 812 (1945).

P. D. Bartlett and R. Altschul, J. Am. Chem. Soc., 67, 816 (1945).

11. A. Matsumoto, A. Okamoto, S. Okuno, and H. Aota, Angew. Makromol. Chem., 240, 275 (1996).

12. A. Matsumoto and M. Oiwa, J. Polym. Sci., A-1, 8, 751 (1970).

13. W. H. Stockmayer, J. Chem. Phys., 12, 125 (1944).

14. M. Gordon, J. Chem. Phys., 22, 610 (1954).

15. A. Matsumoto, H. Nakajima, and M. Oiwa, Netsukokasei Jushi (J. Thermosetting Plastics, Japan), 9, 141 (1988).

16. A. Matsumoto, A. Hamada, and H. Aota, unpublished.

17. A. Matsumoto, S. Yokoyama, T. Khono, and M. Oiwa, J. Polym. Sci., Polym. Phys. Ed., 15, 127 (1977).

18. A. Matsumoto, S. Okuno, and H. Aota, Macromol. Symp., 93, 1 (1995).

19. A. Matsumoto, K. Yokoyama, and H. Aota, Eur. Polym. J., 36, 20 (2000). 